\title{
Epigenetic markers and response to chemotherapy in cancer
}

\author{
Gordon Strathdee* \\ Centre for Oncology and Applied Pharmacology, Cancer Research UK Beatson Laboratories, Glasgow University, \\ Glasgow G61 1BD, UK
}

\begin{abstract}
The last ten years has seen an explosion in interest in epigenetic mechanisms of control of gene expression. This is particularly true in the field of cancer research where epigenetic alterations are now regarded as equally important as genetic alterations in the development and progression of cancer. Of particular interest is altered DNA methylation, which is a key feature of essentially all tumour types. Aberrant methylation of $\mathrm{CpG}$ islands represents an ideal candidate for both diagnostic and prognostic markers in cancer. It is highly prevalent, very largely tumour specific and potentially far more readily detectible than most genetic alterations. This review will discuss the genes already identified as potential epigenetic markers of drug response, as well as the rapidly improving technology for detection of methylation which has greatly expanded the potential sources of tumour specific DNA that can be used for epigenetic marker analysis.
\end{abstract}

Keywords: Epigenetics, drug resistance, chemotherapy, DNA methylation

\section{Introduction}

Cancer has long been regarded as a genetic disease and mutations and deletions of many genes have been shown to be critical in the development of cancer. However, in recent years it has become apparent that epigenetic changes are as important as genetic alterations in the process of tumourigenesis [6]. Epigenetic changes in genes can be defined as a heritable change in gene expression that is not a consequence of a change in the DNA sequence. Such epigenetic changes fall predominantly into two categories - Covalent changes to DNA itself, which is essentially limited to DNA methylation at cytosine residues and secondly modifications of the DNA associated histones, which can take the form of acetylation, methylation, phosphorylation or ubiquitination [4].

\footnotetext{
* Address for correspondence: Gordon Strathdee, Centre for Oncology and Applied Pharmacology, Cancer Research UK Beatson Laboratories, Glasgow University, Switchback Road Glasgow G61 1BD, UK. Fax: +44 141330 4127; E-mail: g.strathdee@ beatson.gla.ac.uk.
}

DNA methylation is the only commonly occurring modification of human DNA and results from the activity of a family of DNA methyltransferase (DNMT) enzymes that catalyse the addition of a methyl group to cytosine residues at $\mathrm{CpG}$ dinucleotides [5]. The human genome exhibits a clear depletion of $\mathrm{CpG}$ dinucleotides, presumably due to the high rate of deamination of 5-methyl cytosine to thymine [5]. However, the genome also contains small stretches, up to a few kilobases in length, which are comparatively rich in $\mathrm{CpG}$ dinucleotides, known as $\mathrm{CpG}$ islands. Unlike the bulk of DNA, where the $\mathrm{CpG}$ dinucleotides are highly methylated, the $\mathrm{CpG}$ dinucleotides in these islands are usually methylation free in adult tissue and this pattern of DNA methylation is stably inherited from one cell generation to the next [5]. The genome consists of around 30,000 CpG islands and 50-60\% of these are associated with genes, usually within the promoter/1st exon region [6]. The functional importance of $\mathrm{CpG}$ islands was revealed by studies demonstrating that methylation of $\mathrm{CpG}$ islands within gene promoters leads to transcriptional repression of the associated gene [5]. 
DNA methylation in particular has recently shown great promise as a marker that can be used to predict outcome or response to therapy in cancer patients and also as a mechanism for early cancer detection. During the last ten years there has been an explosion of research on epigenetic mechanisms of control of gene expression and this has lead to greatly improved methodologies for the detection of epigenetic changes in general and DNA methylation in particular. Consequently alterations in DNA methylation can be detected not only in primary tumour samples, but also in a variety of more readily obtainable samples (such as serum, saliva or urine) which makes DNA methylation a very attractive mechanism for monitoring malignant disease where repeat sampling of the primary lesion would often not be possible. In addition, in contrast to the methylation free status of $\mathrm{CpG}$ islands in normal cells, $\mathrm{CpG}$ island hypermethylation is highly prevalent in all types of human malignancy and up to several thousand $\mathrm{CpG}$ islands can be aberrantly methylated in a single tumour [7]. The highly tumour specific nature of $\mathrm{CpG}$ island hypermethylation means that it can readily be detected even in samples with high levels of contamination with normal DNA.

\section{Hypermethylation of genes and response to chemotherapy}

Genes involved in essentially all facets of tumour development and progression have been shown to be targeted by aberrant methylation in human malignancy [6] and many of these genes, such as those involved in DNA repair or control of apoptosis, have the clear potential to influence the response of cancer cells to therapeutic agents, both by increasing or by decreasing sensitivity (Fig. 1).

\subsection{Genes associated with drug resistance}

CpG island methylation of a number of genes has been linked with poor response to a number of clinically useful chemotherapeutic drugs. Probably the clearest example is the mismatch repair (MMR) protein MLH1. In addition to its role in repair of normally occurring polymerase errors during DNA replication, the MMR system plays a key role in recognition and response to DNA damage induced by a number of commonly used therapeutic drugs [15]. However, recognition of such damage by the MMR system leads not to DNA

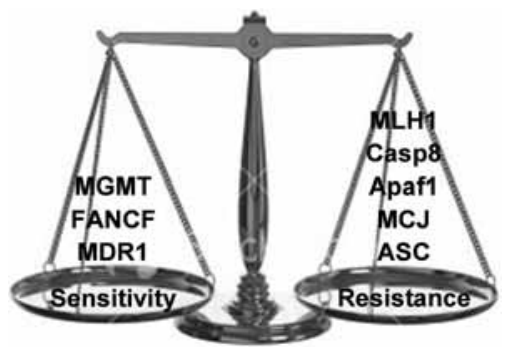

Fig. 1. Epigenetic markers of increased drug sensitivity and resistance. Hypermethylation of the MGMT, FANCF and MDR1 genes have been reported to correlate with increased sensitivity to chemotherapeutic drugs, whilst hypermethylation of MLH1, CASP8, APAF1, MCJ and ASC [24] has been associated with drug resistance.

repair but initiates apoptotic signals through a variety of pathways, including activation of p53 and p73 [24].

Loss of MLH1 expression is common in colorectal, gastric, endometrial and ovarian cancers, and while mutations of this gene are rarely found, in the overwhelming majority of cases loss of expression is related to hypermethylation of the MLH1 CpG island. In ovarian cancer cell lines selection for resistance to cisplatin results in loss of MLH1 expression due to promoter hypermethylation [32]. Reversal of this methylation, using an inhibitor of the DNMT enzymes, results in re-expression of MLH1 and re-sensitisation of the cells to cisplatin, using both in vitro and in vivo models $[27,32]$. Similar experiments have also shown that reversal of MLH1 CpG island methylation in colorectal cancer cells results in re-sensitisation to 5-fluorouracil (5-FU) [2].

Clinical studies also confirm the potential utility of MLH1 methylation as a predictive marker for response to chemotherapy. In ovarian cancer assessment of MLH1 methylation in tumour derived DNA, isolated from serum samples at relapse, identified a much higher rate of MLH1 methylation following chemotherapy than before therapy (33\% versus 12\%) and MLH1 promoter methylation was associated with poor survival [19]. Similarly, in colorectal cancer, tumours exhibiting microsatellite instability, the hallmark of loss of MMR activity and almost always due to MLH1 promoter methylation [8], were found to receive no benefit from 5-FU based treatment, whereas patients lacking MSI had clearly improved survival following 5-FU therapy [28]. These clinical studies confirm the original in vitro studies suggesting that MLH1 methylation is an important determinant of chemosensitivity in cancer cells.

As shown in Table 1 methylation of a number of other genes has been shown to be associated with poor 
response to a number of different therapeutic agents. Many of these play important role in the process of apoptosis or programmed cell death. For example methylation of Apaf1, which is a key downstream effector of p53 induced apoptosis [30], in association with loss of heterozygosity results in loss of expression in malignant melanoma samples and cell lines and reexpression following reversal of DNA methylation results in increased sensitivity to adriamycin [31]. Similarly Caspase-8, a key downstream effector of TRAIL mediated apoptosis, has been shown to be frequently hypermethylated in many tumour types, particularly neuronal tumours. Loss of Caspase 8 has been shown to be a poor prognostic factor in medulloblastoma [26] and re-expression of Caspase8 restores sensitivity of neuronal cancer cells to TRAIL ligand [18]. Other genes whose functions are less clear may also prove useful as markers for chemoresponse or survival. For example the MCJ gene, a member of the DNAJ protein family of unknown function, was found to increase sensitivity to a number of chemotherapeutic agents in vitro [29] and high MCJ methylation levels correlate both with response to therapy and overall survival in ovarian cancer [33].

\subsection{Genes associated with drug sensitivity}

The tumour specificity of most $\mathrm{CpG}$ island hypermethylation events makes DNA methylation a very attractive therapeutic target, both in primary tumours, but also for the reversal of clinical drug resistance. However not all methylation events that influence drug sensitivity in tumours leads to resistance and indeed the clearest example of a methylated gene that predicts response to chemotherapy is the MGMT gene, methylation of which is associated with improved response to alkylating agents [13]. MGMT is a DNA repair enzyme that repairs adducts that can be induced by a number of therapeutically useful alkylating agents and levels of MGMT have long been known to influence sensitivity to such agents [13]. MGMT methylation was first linked to drug sensitivity in 1997 by Watts and co-workers [36]. And a few years later MGMT methylation was demonstrated to be a highly significant predictor of survival in glioma [12]. This observation has since been confirmed in multiple studies, mainly in neurological tumours but also in lymphoma [11]. In addition, the utility of MGMT hypermethylation as a prognostic factor has been confirmed in a prospective clinical trial which demonstrated dramatic differences in survival $(62 \%$ for methylated patients versus $8 \%$ in unmethylated patients at 18 months) [21].

Although methylation of other genes has also been associated with drug sensitivity the evidence is somewhat more conflicting than that of MGMT. Numerous reports have investigated the relationship of methylation of the MDR1 gene, which encodes the Pglycoprotein (P-gp) efflux pump, and drug resistance and indeed many of these have suggested an inverse relation ship between MDR1 methylation and expression of P-gp, with increased methylation being associated with reduced P-gp expression and consequent increase in sensitivity to chemotherapeutic drugs which would be substrates for the pump (reviewed in [23]. However, other reports have found no association between methylation of the gene and expression even in cell lines [17] and at least one report found that methylation even increased MDR1 expression, through suppression of a repressor element [1]. Overall it remains unclear if methylation does play a significant role in suppression of MDR1 expression in cancer. Similarly a recent report identified a correlation between methylation of the FANCF gene, which plays a role in repair of double strand DNA breaks, and chemoresistance in ovarian cancer [34]. However, this report failed to demonstrate high levels of methylation of this gene in primary tumours and a subsequent investigation of FANCF methylation in over 100 ovarian tumours failed to identify any evidence of FANCF methylation [35], suggesting that methylation of FANCF is unlikely to play a significant role in the chemosensitivity of ovarian cancer.

\section{Detection of methylation}

One of the most attractive features of DNA methylation as a marker for drug resistance, and indeed other aspects of tumourigenesis is the wide array of technologies now available for detection of methylation. These include methods that allow for very sensitive detection of single gene methylation, making it possible to detect aberrant methylation in a wide variety of sample types including many which are readily obtainable, including serum, sputum and urine, allowing methylation to be readily used to monitor changes in tumour biology over the course of treatment. In addition, technologies are now available for array based detection of methylation at high numbers of $\mathrm{CpG}$ islands, enabling for methylation profiling of tumours. 


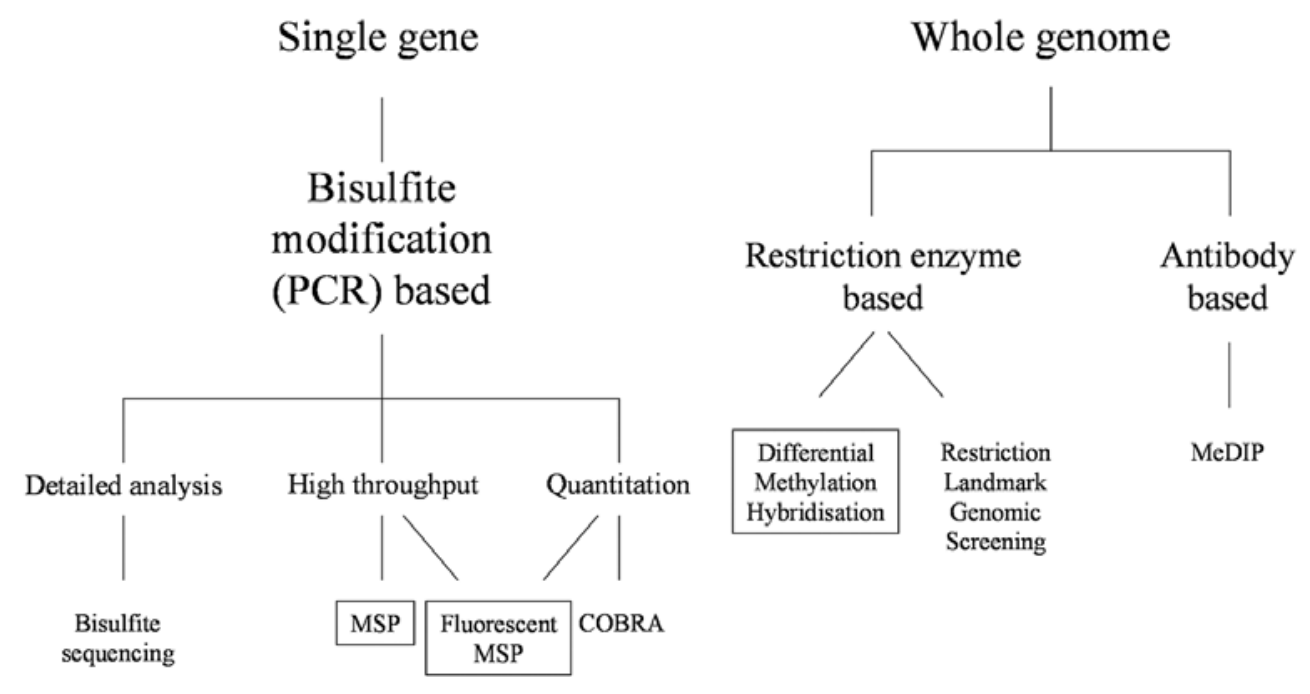

Fig. 2. Commonly used methods for the analysis of $\mathrm{CpG}$ island DNA methylation. A flow diagram of commonly used methods for the analysis of single gene and genome wide methylation, detailing the mechanisms by which differential methylation is detected and (for the single gene methods) their primary technical advantages. Boxed techniques are those that have been used for studies on epigenetic markers of drug resistance. MeDIP, Methylation analysis by DNA immunoprecipitation [36].

Detection of genomic DNA methylation was initially carried out primarily through the use of methylation sensitive restriction enzymes [9]. These enzymes will only cut DNA at their recognition sequences when the DNA in not methylated and therefore the failure of the methylation sensitive enzymes to digest DNA could be taken as evidence of methylation. However, as detection was usually carried out by Southern blotting such analysis was greatly limited by the large amounts of DNA needed to assess even a single $\mathrm{CpG}$ site. However, analysis of methylation was revolutionised by the introduction of sodium bisulfite modification (Fig. 2). Treatment of genomic DNA with sodium bisulfite leads to the conversion of cytosine residues to uracils (and then to thymidine in subsequent PCR amplification). However, methylated cytosines remain unaffected by bisulfite treatment and consequently a sequence difference is produced in which methylated $\mathrm{CpGs}$ remain but unmethylated $\mathrm{CpGs}$ are converted to TpG. This was initially used for bisulfite sequencing [16] (Fig. 2), and was further adapted through the use of methylation specific PCR (MSP) which allowed for greatly increased throughput [22] and through COBRA analysis [39] (Fig. 2) which allows for rapid quantitation of methylation levels.

Through the use of these PCR based assays, in particular MSP, it is now possible to detect aberrant tumour associated methylation changes in a variety of samples in which only very small quantities of tumour derived DNA are present. These include serum [14], urine [20], sputum [3] and many others. Using such samples greatly expands the potential uses of DNA methylation detection for monitoring of treatment, early detection and potentially even large screening programs, where access to primary tumour samples is not practical, although the sensitivity of detection is clearly reduced

\subsection{Single gene versus array technology}

In the above discussion on genes whose methylation effects drug sensitivity all the analysis was done using approaches that analyse only a single gene. While such approaches can clearly identify markers which may alter the response of tumour cells to treatment, it is clearly the case that tumour cell response to therapeutic drugs will be a multigenic trait and that analysis of single genes is unlikely to provide precise information about potential response to therapy. A number of approaches have now been reported which allow genome wide analysis of $\mathrm{CpG}$ island methylation status (Fig. 2) and these provide the possibility of using a more global approach to the use of methylation to predict response. However, to date only a single study has addressed this question. This study used differential methylation hybridisation to assess the methylation status of nearly $8000 \mathrm{CpG}$ islands in ovarian tumours and demonstrated a very striking correlation between high methylation levels and poor response to treatment [38]. Clearly such powerful techniques have great potential for the prediction of chemosensitivity in human cancer, 
however they also have clear drawback compared to the single gene approach. Firstly, such techniques are far more technically challenging and require specialist expertise and equipment, making them unsuitable for general laboratory use. Secondly, the amount and quality of DNA required could only be obtained by direct sampling of the tumour, making them much more limited in their potential application than the single gene approaches.

\subsection{Quantitation versus qualitative}

By far the most frequently used technique for both methylation analysis in general and for identification of chemotherapeutic drug response makers is MSP. While this technique has excellent sensitivity for detection of the presence or absence of methylation at a single target gene, it provides only qualitative data. Thus positives obtained with this technique give no indication as to whether the identified methylation is present in all tumour cells or just a tiny fraction. This clearly has the potential to alter the significance of the identified methylation. For example, MGMT methylation has been clearly linked to improved response to a number of therapeutic agents, presumably due to the importance of this enzyme during repair of the induced DNA damage. However, if methylation was only present in a tiny fraction of cells then such improved response would not be predicted. Also while methylation of the MCJ gene was found to correlate with response to therapy in ovarian cancer, this was only true of tumours with very high levels of methylation and this association would have been missed without the use of quantitative analysis [33]. Both the use of COBRA assays and fluorescent PCR based MSP assays [10] have the potential to overcome this limitation while maintaining the comparative ease of use of the MSP assay. However, to date their potential use in the prediction of chemoresponse in tumours has not been widely tested, but it is likely the use of such assays could further improve the potential of methylation based makers for prediction of response.

\section{Conclusions}

The prevalence, high degree of tumour specificity and comparative ease of detection of aberrant $\mathrm{CpG}$ island methylation makes such alterations very attractive candidates for use as markers of response to therapy in cancer patients. Already a number of markers are known to be associated with both improved and poor response to specific cancer therapies. This list will undoubtedly grow substantially in the upcoming years, however appropriately sized clinical studies of such markers will be required to clearly demonstrate their potential use in the clinic. In addition, appropriate methodologies will have to be utilised if the potential of these markers is going to be fully realised. Studies will have to be performed integrating both quantitative data at particular loci as well as the use of multiple epigenetic markers to get a more accurate picture of potential response to therapy. Furthermore the potential of methods for genome wide assessment of altered methylation clearly merits much further study to determine if it could complement, or potentially replace, single loci markers. Lastly, the potential reversibility of epigenetic alterations make them ideal candidates for modulation of tumour cell response to chemotherapy. Studies have already shown for several of the identified markers, that reversal of aberrant DNA methylation can lead to re-sensitisation of tumour cells to therapy. Thus the epigenetic markers identified may also represent potential therapeutic targets for strategies that combine conventional chemotherapeutics with epigenetic based therapies, such as the DNA methylation inhibitor Decitabine. The potential of such therapeutic approaches is already being tested in early phase clinical trials.

\section{Acknowledgements}

This work was supported by a Cancer Research UK programme grant awarded to Prof. Robert Brown.

\section{References}

[1] T. Ando, M. Nishimura and Y. Oka, Decitabine (5-Aza-2'deoxycytidine) decreased DNA methylation and expression of MDR-1 gene in K562/ADM cells, Leukemia 11 (2000), 1915-1920.

[2] C. N. Arnold, A. Goel and C.R. Boland, Role of hMLH1 promoter hypermethylation in drug resistance to 5-fluorouracil in colorectal cancer cell lines, Int J Cancer 106 (2003), 66-73.

[3] S.A. Belinsky, K.J. Nikula, W.A. Palmisano, R. Michels, G. Saccomanno, E. Gabrielson, S.B. Baylin and J.G. Herman, Aberrant methylation of p16(ink4a) is an early event in lung cancer and a potential biomarker for early diagnosis, Proc Natl Acad Sci USA 20 (1998), 11891-11896.

[4] S.L. Berger, Histone modifications in transcriptional regulation, Curr Opin Genet Dev 2 (2002), 142-148.

[5] A. Bird, The Relationship of DNA Methylation to Cancer, Cancer Surveys (1996), 87-101. 
[6] J. Costello, F and C. Plass, Methylation matters, J Med Genet (2001), 285-303.

[7] J.F. Costello, M.C. Fruhwald, D.J. Smiraglia, L.J. Rush, G.P. Robertson, X. Gao, F.A. Wright, J.D. Feramisco, P. Peltomaki, J.C. Lang, D.E. Schuller, L. Yu, C.D. Bloomfield, M.A. Caligiuri, A. Yates, R. Nishikawa, H.S. Huang, N.J. Petrelli, X. Zhang, M.S. O'Dorisio, W.A. Held, W. Cavenee and C. Plass, Aberrant CpG-island methylation has non-random and tumour-type-specific patterns, Nat Genet (2000), 132-138.

[8] J.M. Cunningham, E.R. Christensen, D.J. Tester, C.Y. Kim, P.C. Roche, L.J. Burgart and S.N. Thibodeau, Hypermethylation of the hMLH1 promoter in colon cancer with microsatellite instability, Cancer Res 15 (1998), 3455-3460.

[9] C. Dahl and P. Guldberg, DNA methylation analysis techniques, Biogerontology 4 (2003), 233-250.

[10] C.A. Eads, K.D. Danenberg, K. Kawakami, L.B. Saltz, C. Blake, D. Shibata, P.V. Danenberg and P.W. Laird, MethyLight: a high-throughput assay to measure DNA methylation, Nuc Acid Res 8 (2000), E32.

[11] M. Esteller, G. Gaidano, S.N. Goodman, V. Zagonel, D. Capello, B. Botto, D. Rossi, A. Gloghini, U. Vitolo, A. Carbone, S.B. Baylin and J.G. Herman, Hypermethylation of the DNA repair gene $\mathrm{O}(6)$-methylguanine DNA methyltransferase and survival of patients with diffuse large B-cell lymphoma, $J$ Natl Cancer Inst 1 (2002), 26-32.

[12] M. Esteller, J. Garcia-Foncillas, E. Andion, S.N. Goodman, O.F. Hidalgo, V. Vanaclocha, S.B. Baylin and J.G. Herman, Inactivation of the DNA-repair gene MGMT and the clinical response of gliomas to alkylating agents, $N$ Engl J Med 19 (2000), 1408-1409.

[13] M. Esteller and J.G. Herman, Generating mutations but providing chemosensitivity: the role of O6-methylguanine DNA methyltransferase in human cancer, Oncogene 1 (2004), 1-8.

[14] M. Esteller, M. Sanchez-Cespedes, R. Rosell, D. Sidransky, S.B. Baylin and J.G. Herman, Detection of aberrant promoter hypermethylation of tumour suppressor genes in serum DNA from non-small cell lung cancer patients, Cancer Res (1999), 67-70.

[15] D. Fink, S. Aebi and S.B. Howell, The role of DNA mismatch repair in drug resistance, Clin Cancer Res (1998), 1-6.

[16] M. Frommer, L.E. McDonald, D.S. Millar, C.M. Collis, F. Watt, G.W. Grigg, P.L. Molloy and C.L. Paul, A genomic sequencing protocol that yields a positive display of 5methylcytosine residues in individual DNA strands, Proc Natl Acad Sci USA 5 (1992), 1827-1831.

[17] K.B. Fryxell, S.B. McGee, D.K. Simoneaux, C.L. Willman and M.M. Cornwell, Methylation analysis of the human multidrug resistance 1 gene in normal and leukemic hematopoietic cells, Leukemia 6 (1999), 910-917.

[18] S. Fulda, M.U. Kufer, E. Meyer, F. van Valen, B. DockhornDworniczak and K.M. Debatin, Sensitization for death receptor- or drug-induced apoptosis by re-expression of caspase- 8 through demethylation or gene transfer, Oncogene 41 (2001), 5865-5877.

[19] G. Gifford, J. Paul, P.A. Vasey, S.B. Kaye and R. Brown, The acquisition of hMLH1 methylation in plasma DNA after chemotherapy predicts poor survival for ovarian cancer patients, Clin Cancer Res 13 (2004), 4420-4426.

[20] C. Goessl, H. Krause, M. Muller, R. Heicappell, M. Schrader, J. Sachsinger and K. Miller, Fluorescent methylation-specific polymerase chain reaction for DNA-based detection of prostate cancer in bodily fluids, Cancer Res 21 (2000), 59415945.
[21] M.E. Hegi, A.C. Diserens, S. Godard, P.Y. Dietrich, L. Regli, S. Ostermann, P. Otten, G. Van Melle, N. de Tribolet and R. Stupp, Clinical trial substantiates the predictive value of $\mathrm{O}$ 6-methylguanine-DNA methyltransferase promoter methylation in glioblastoma patients treated with temozolomide, Clin Cancer Res 6 (2004), 1871-1874.

[22] J.G. Herman, J.R. Graff, S. Myohanen, B.D. Nelkin and S.B. Baylin, Methylation-specific PCR: A novel PCR assay for methylation status of CpG islands, Proc Natl Acad Sci USA (1996), 9821-9826.

[23] P. Kantharidis, S. El-Osta, M. Silva, G. Lee, X.F. Hu and J. Zalcberg, Regulation of MDR1 gene expression: emerging concepts, Drug Resist Updat 2 (2000), 99-108.

[24] G.M. Li, The role of mismatch repair in DNA damage-induced apoptosis, Oncol Res 9 (1999), 393-400.

[25] T. Ohtsuka, X.F. Liu, Y. Koga, Y. Kitajima, Y. Nakafusa, C.W. Ha, S.W. Lee and K. Miyazaki, Methylation-induced silencing of ASC and the effect of expressed ASC on p53-mediated chemosensitivity in colorectal cancer, Oncogene (2005).

[26] C. Pingoud-Meier, D. Lang, A.J. Janss, L.B. Rorke, P.C. Phillips, T. Shalaby and M.A. Grotzer, Loss of caspase- 8 protein expression correlates with unfavorable survival outcome in childhood medulloblastoma, Clin Cancer Res 17 (2003), 6401-6409.

[27] J.A. Plumb, G. Strathdee, J. Sludden, S.B. Kaye and R. Brown, Reversal of drug resistance in human tumour xenografts by 2'deoxy-5-azacytidine-induced demethylation of the hMLH1 gene promoter, Cancer Res (2000), 6039-6044.

[28] C.M. Ribic, D.J. Sargent, M.J. Moore, S.N. Thibodeau, A.J. French, R.M. Goldberg, S.R. Hamilton, P. Laurent-Puig, R. Gryfe, L.E. Shepherd, D. Tu, M. Redston and S. Gallinger, Tumor microsatellite-instability status as a predictor of benefit from fluorouracil-based adjuvant chemotherapy for colon cancer, N Engl J Med 3 (2003), 247-257.

[29] V. Shridhar, B. K.C., J. Staub, R. Avula, Y.K. Lee, K. Kalli, H. Huang, L.C. Hartmann, S.H. Kaufmann and D.I. Smith, Loss of Expression of a New Member of the DNAJ Protein Family Confers Resistance to Chemotherapeutic Agents Used in the Treatment of Ovarian Cancer, Cancer Res (2001), 4258-4265.

[30] M.S. Soengas, R.M. Alarcon, H. Yoshida, A.J. Giaccia, R. Hakem, T.W. Mak and S.W. Lowe, Apaf-1 and caspase-9 in p53-dependent apoptosis and tumor inhibition, Science $\mathbf{5 4 1 1}$ (1999), 156-159.

[31] M.S. Soengas, P. Capodieci, D. Polsky, J. Mora, M. Esteller, X. Opitz-Araya, R. McCombie, J.G. Herman, W.L. Gerald, Y.A. Lazebnik, C. Cordon-Cardo and S.W. Lowe, Inactivation of the apoptosis effector Apaf-1 in malignant melanoma, Nature 6817 (2001), 207-211.

[32] G. Strathdee, M. MacKean, M. Illand and R. Brown, A role for methylation of the $h M L H 1$ promoter in loss of hMLH1 expression and drug resistance in ovarian cancer, Oncogene (1999), 2335-2341.

[33] G. Strathdee, J.K. Vass, K.A. Oien, N. Siddiqui, J. CurtoGarcia and R. Brown, Demethylation of the MCJ gene in stage III/IV epithelial ovarian cancer and response to chemotherapy, Gynecol Oncol 3 (2005), 898-903.

[34] T. Taniguchi, M. Tischkowitz, N. Ameziane, S.V. Hodgson, C.G. Mathew, H. Joenje, S.C. Mok and A.D. D'Andrea, Disruption of the Fanconi anemia-BRCA pathway in cisplatinsensitive ovarian tumors, Nat Med 5 (2003), 568-574.

[35] J.M. Teodoridis, J. Hall, S. Marsh, H.D. Kannall, C. Smyth, J. Curto, N. Siddiqui, H. Gabra, H.L. McLeod, G. Strathdee and $\mathrm{R}$. Brown, $\mathrm{CpG}$ island methylation of DNA damage response 
genes in advanced ovarian cancer, Cancer Res 19 (2005), 8961-8967.

[36] G.S. Watts, R.O. Pieper, J.F. Costello, Y.M. Peng, W.S. Dalton and B.W. Futscher, Methylation of discrete regions of the O6-methylguanine DNA methyltransferase (MGMT) CpG island is associated with heterochromatinization of the MGMT transcription start site and silencing of the gene, Mol Cell Biol 9 (1997), 5612-5619.

[37] M. Weber, J.J. Davies, D. Wittig, E.J. Oakeley, M. Haase, W.L. Lam and D. Schubeler, Chromosome-wide and promoterspecific analyses identify sites of differential DNA methy- lation in normal and transformed human cells, Nat Genet $\mathbf{8}$ (2005), 853-862.

[38] S.H. Wei, C.M. Chen, G. Strathdee, J. Harnsomburana, C.R. Shyu, F. Rahmatpanah, H. Shi, S.W. Ng, P.S. Yan, K.P. Nephew, R. Brown and T.H. Huang, Methylation microarray analysis of late-stage ovarian carcinomas distinguishes progression-free survival in patients and identifies candidate epigenetic markers, Clin Cancer Res 7 (2002), 2246-2252.

[39] Z. Xiong and P.W. Laird, COBRA: a sensitive and quantitative DNA methylation assay, Nuc Acid Res (1997), 2532-2534. 


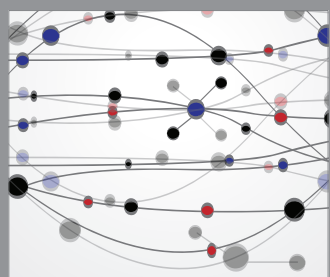

The Scientific World Journal
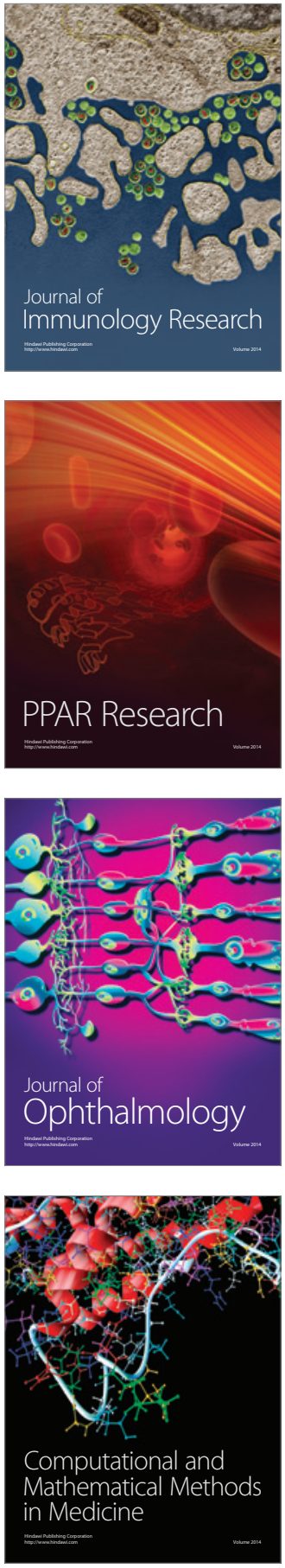

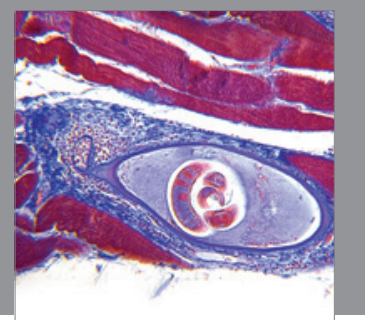

Gastroenterology

Research and Practice
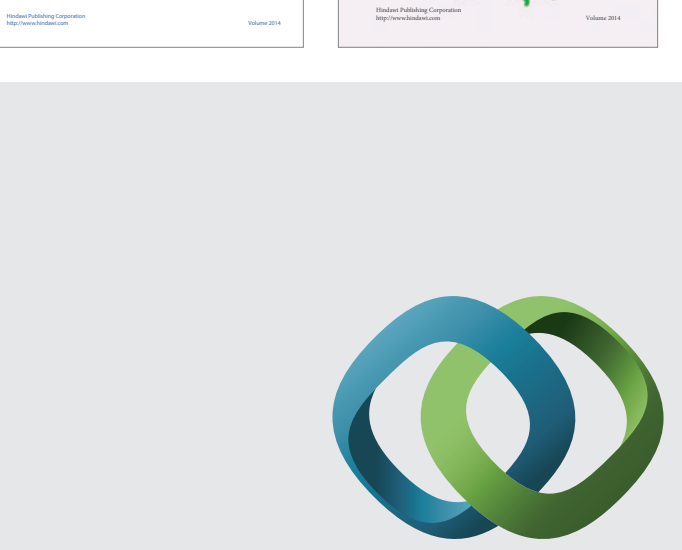

\section{Hindawi}

Submit your manuscripts at

http://www.hindawi.com
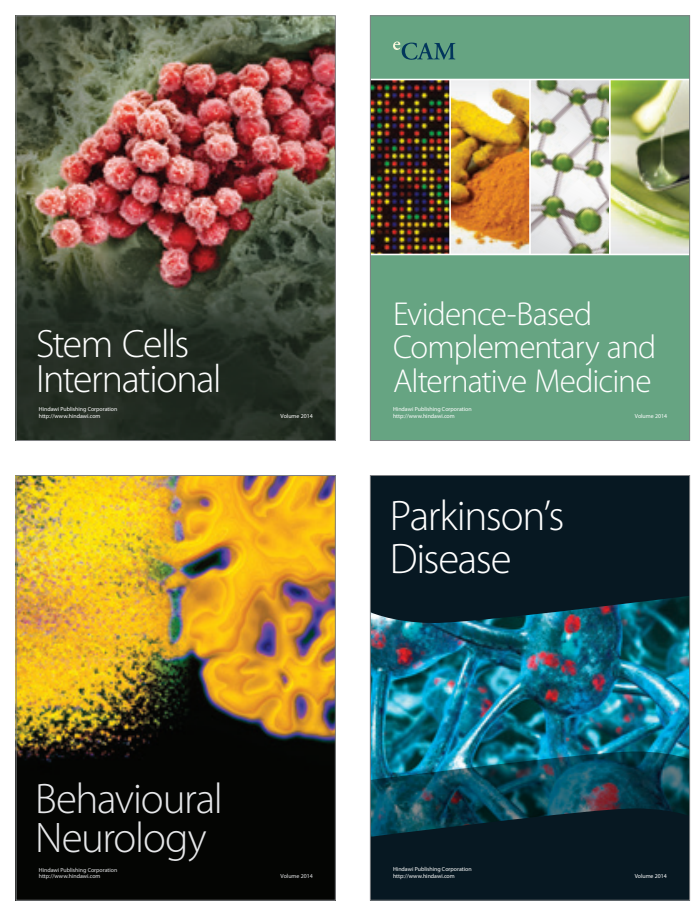

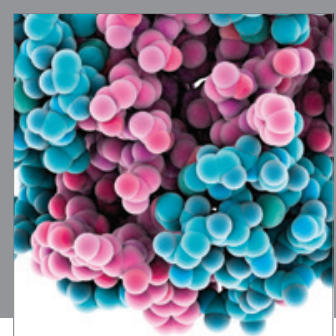

Journal of
Diabetes Research

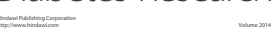

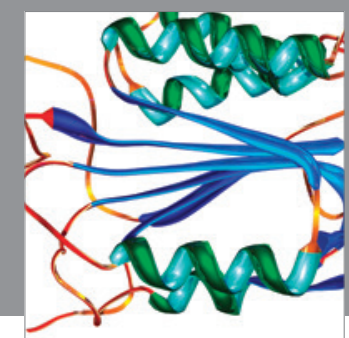

Disease Markers
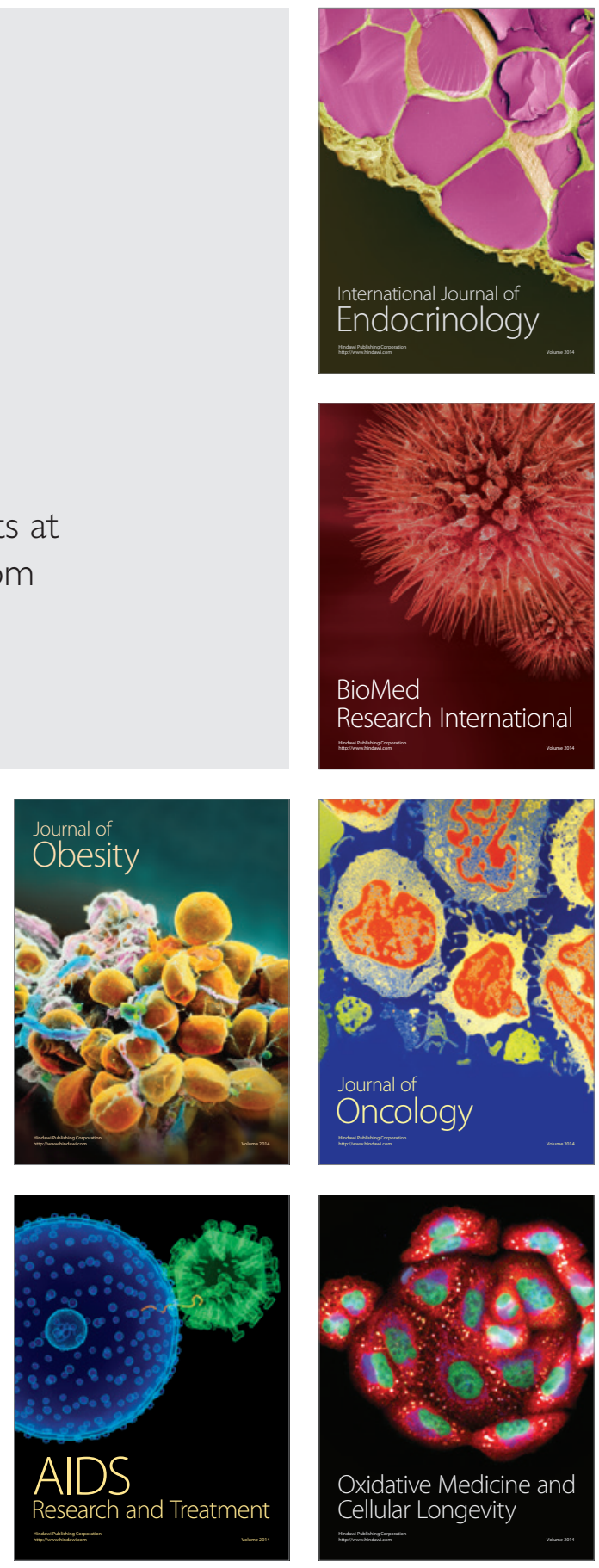